\title{
STERLING: A framework for serious games to encourage recycling
}

\author{
Ester Diego $^{\mathrm{a}}$, David Carravilla ${ }^{\mathrm{b}}$, Guillermo Vicente ${ }^{\mathrm{b}}$, Hector Del-Campo Pando ${ }^{\mathrm{a}}$, Daniel \\ Barba $^{\mathrm{a}}$, Diego R. Llanos ${ }^{\mathrm{a}, \mathrm{b}}$, and Juan A. March ${ }^{\mathrm{b}}$ \\ ${ }^{\text {a}}$ Dpto. Informática, Universidad de Valladolid, Valladolid, Spain \\ ${ }^{\mathrm{b}}$ RDNest, Valladolid, Spain
}

\begin{abstract}
Waste disposal and recycling is becoming one of the main problems in Western countries. Improving both recycling culture among citizenship and waste collection and treatment logistics is critical to augment the percentage of waste being recycled. In this paper we present STERLING, an initiative that aims to help in both fields. STERLING is a framework composed by a low-cost, low-energy sensor installed in recycling containers to measure fill level and other physical parameters. The sensor is activated magnetically each time the container lid is opened by a user. Instead of directly sending this information to a cloud-based server, our sensor broadcasts a Bluetooth Low Energy (BLE) packet to the surrounding area. An App running in the mobile phone of the user performs two actions: To capture this information, re-sending it to the cloud-based server, and to assign credit to this particular user for having used the recycling container. In this way, users are rewarded for using the container, and the infrastructure benefits from cost-free communications from the container to the server. In this paper we will describe our idea in detail, showing how it can be used to develop a rewarding schema that encourages recycling.
\end{abstract}

Keywords: Waste disposal, recycling, low-cost sensors, low-energy sensors, gamification, IoT

\section{INTRODUCTION}

Waste disposal is one of the main problems faced by advanced societies. Modern lifestyle makes extensive use of packaging material, that should be separated and recycled appropriately ${ }^{1}$. To improve recycling habits among citizenship, some kind of rewarding mechanism would be useful. The basic mechanism, to re-buy empty bottles or cans, has been widely used during the past century. However, this effective mechanism has been progressively abandoned, partly because of the low involvement of shops and producers with recycling activities. Nowadays, most European cities have containers designed specifically to hold empty cans and bottles. The problem now is how to encourage citizenship to use them.

These containers poses an additional challenge, in this case related with their logistic. To make recycling a more attractive activity, local authorities should empty recycling containers promptly. However, in many cases containers become suddenly full and waste is abandoned nearby, degrading the aspect of the cities and harming all the efforts devoted to encourage recycling. During the last years, several companies have developed filling sensors, in order to detect these situations and to improve waste collection routes and logistic. However, these devices need a working Internet connection to transmit the information to the recycling company, a solution that is expensive both in terms of energy needed by the device, and in terms of the cost of the Internet connection, usually carried out using a SIM modem that imposes a fixed, monthly cost to the company.

In this paper we propose a solution to both encourage recycling habits among citizenship and solve the problem of detecting and managing full containers without incurring in the costs mentioned above. Our proposal, called STERLING, relies on a relatively simple device that measure some physical magnitudes of the container, including fill level, temperature and humidity. Our device is usually in "sleep" mode, thus consuming just a few micro-amperes per hour, and is waken up each time the lid of the container is open. At that time, our device

For both technological request and business proposals, please contact Dr. Diego R. Llanos, RDNest, Edificio CTTA, Paseo Belén 9A, 47011 Valladolid, Spain. e-mail: diego@rdnest.com. 

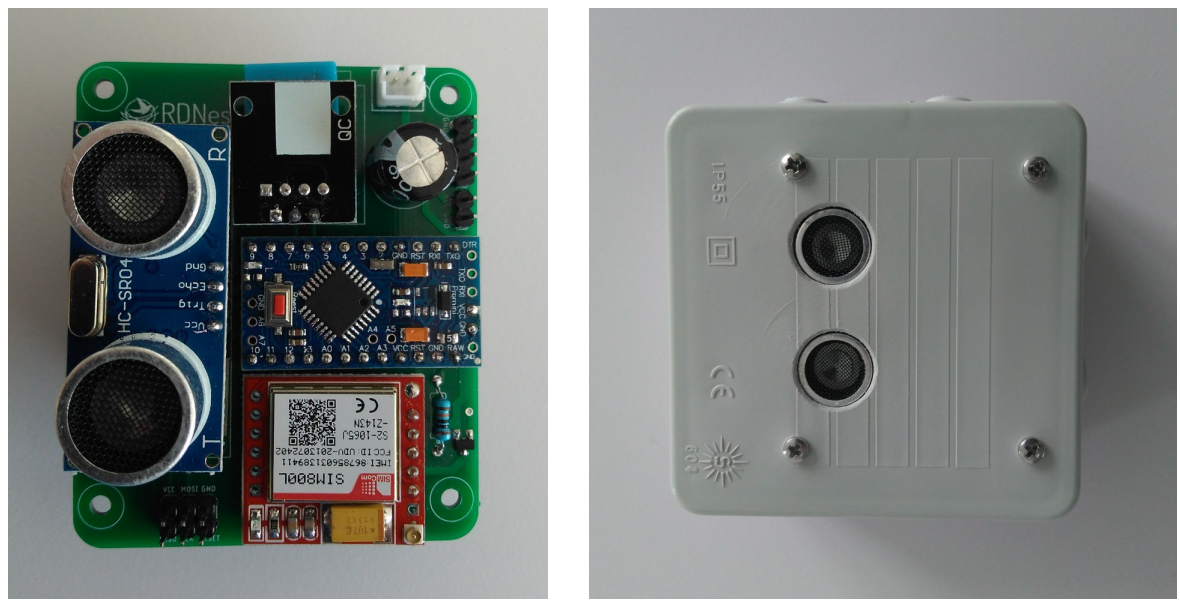

Figure 1. Autonomous sensor system for recycling containers developed by RDNest, that includes a SIM modem (left) and external packaging (right).

measures the state of the container, and broadcast a Bluetooth Low Energy (BLE) ${ }^{2}$ packet with this information. An application installed by the citizen in their mobile phone is then used to (a) retransmit this information to the centralized server, and (b) give credit to the citizen for using the recycling container. In this way we solve both problems: To instrument containers with a low-cost, low-energy device that has no communication running costs, and to reward citizenship involved in recycling activities.

This paper is organized as follows. Section 2 describes some previous efforts to augment citizenship's recycling habits. Section 3 examines the design space of sensoring solutions. Section 4 describes the STERLING sensor in detail. Section 5 shows some design guidelines for the STERLING accompanying app. Section 6 discusses how the STERLING framework can be used to learn more about users' habits and encourage recycling. Finally, Sect. 7 concludes the paper.

\section{RELATED WORK}

During the last years, there is an increasing interest on taking advantage of Internet of Things (IoT) technology to monitor the status of waste containers. Giacobbe et al. ${ }^{3}$ have discussed the need for new smart waste management systems, presenting a comprehensive state of the art on the use of IoT technologies for smart waste recycling. Lindström et $a l .{ }^{4}$ have studied how to optimize recycling management in terms of emptying recycling containers, thus supporting sustainability of natural resources and the circular economy. Chin et al. ${ }^{5}$ have discussed the issues relative to the use of household waste management system equipped with IoT technology. Gattim et $a l .{ }^{6}$ have recently proposed to augment solid waste containers with sensors that measure their working conditions and send this information to the Internet. Measurements not only include fill level, but also the level of potentially-hazardous gases produced by the waste being stored. Other uses of IoT technologies include the use of special sensors to store dry and wet waste in their corresponding underground containers. ${ }^{7}$

Gamification techniques ${ }^{8}$ is a technique to involve people in certain tasks as it were a game, taking advantage of emotional and/or physical rewarding. Regarding gamification techniques to encourage recycling, GonzálezBriones et $a l .{ }^{9}$ have recently proposed a multi agent-based system that encourages citizen participation, using tax reductions as incentives. Other proposals related to waste management include to reward citizens with emoticons and sounds ${ }^{10}$, and to use gamification techniques to improve environmental knowledge and behavior ${ }^{11}$.

\section{DESIGN SPACE OF FILL SENSORS}

IoT technologies offer a myriad of low-cost sensors that can be integrated to monitorize recycling containers. Available sensors allow to measure temperature and humidity, fill level, precise localization using GPS, detection of potentially-hazardous gases, etc. To design an electronic circuit to integrate and manage these sensors is 
straightforward. However, there are two problems that should be solved in order to develop a cost-effective sensor system for this particular application.

The most important one is how to manage communications. It is not enough for the sensor system to collect information related to physical magnitudes. The system should also be able to transmit this information to a centralized server, in order to take advantage of it. In modern societies, mobile phone coverage is almost ubiquous, so the use of modems with SIM cards capable of connecting to Internet is a natural choice. These modems cost a few euros, and communication costs are continuously falling. However, although inexpensive, communication is not for free. Telephone carriers usually charge a certain monthly amount for having an Internet connection, regardless of the fact that the amount of data transmitted from a recycling container is really low. Sending a 200-bytes data packet four times per day, the required bandwidth is just $24 \mathrm{~Kb}$ per month. Even taking into account economy of scale, the use of SIM cards to transmit this information is expensive with respect of the amount of data being effectively transmitted.

Communication costs is not the only drawback to the use of SIM modems to connect the container with the outside world. Another problem is energy consumption. Widely-used modems, such as the SIM800 modem ${ }^{12}$, requires peaks of current of around $2 \mathrm{~A}$. Putting this current into perspective, a sensor system in sleep mode consumes a few micro-amps, and to trigger physical sensors to gather the desired information may consume a few mili-amps. The use of SIM modems requires in turn to equip the sensor system with batteries capable of delivering these peaks of current, and/or the use of not-negligible capacitors. It is also necessary to include batteries capable to store the energy needed for the recycling container during their entire working life (estimated in around five years). To develop a sensor system with these constraints is still possible, and there are some examples in the market. For example, Fig. 1 shows a sensor system, developed by RDNest, that includes a SIM modem. As we will see in the following sections, the STERLING proposal greatly simplifies these requirements.

Finally, a remaining problem is how to link the recycling containers with their end users. This link is critical to reward citizens individually for their recycling habits. There exists a myriad of biometric recognition systems. Some of them requires cameras, footprint readers, or other data-acquisition devices, that can not be fit within the economic (few tens of euros) and energy (mili-amps) constrains of this application. Another possibility is to encourage users to install a mobile App that sends some data to the container. In our opinion, this is a much better solution. Our proposal takes advantage of such a communication, but, as we will see, in reverse order.

\section{THE STERLING SENSOR}

The STERLING sensor includes the following components:

- A low-power CMOS 8-bit microcontroller (ATmega328/P).

- A distance sensor used to measure distance between the waste level and the ceiling of the container (HCSR04).

- A sensor to measure temperature and humidity (DHT11).

- A Reed-type, magnetic switch to detect when the container lid has been opened.

- A Bluetooth 4.0 serial wireless module (CC2541).

- Auxiliary electronics to avoid sensors to consume power when the microcontroller is in sleep mode.

Other digital sensors and devices can be integrated as well, including a GPS module such as the Adafruit Ultimate Breakout, in case the position of the container should be periodically checked.

The behavior of the sensor system is simple. Each time the container lid is opened, the magnetic switch raise an interrupt that wakes up the microcontroller. The microcontroller waits X seconds (to allow the user to deposit the waste), and then measures the distance between the container ceiling and the waste level, temperature and humidity, and any other desirable physical magnitude. Then, instead of sending the information to a cloudbased server using a SIM modem, the STERLING system broadcast a Bluetooth Low Energy packet using 
the Eddystone protocol. The operational range can be determined by adjusting the intensity of the signal being broadcasted, and ranges between 30 and 50 meters. The information broadcasted includes the following information:

- A timestamp.

- An unique identification of the container.

- The telemetry values acquired by the sensors.

- The voltage level measured in the microcontroller, to allow preventive maintenance if the battery is running out of energy.

- The number of times the lid has been opened since the container was originally installed.

As we will see below, the opening counter is an useful feature that allows the recycling organization to acquire knowledge about the use of the container. This counter not only allows to know how frequently the lid is opened, but also to estimate the penetration rate of the STERLING App, an issue discussed in the following section.

The information sent can be optionally encrypted using a public-key mechanism if necessary.

\section{THE STERLING APP}

The second component of the STERLING framework is its App. This App allows a user to sign up, asking for a valid e-mail address, and includes a background service that listens for BLE packets coming from recycling containers. Once such a packet is received, the App simply retransmit this information to a cloud-based server. The information being sent includes:

- All the information gathered from the recycling container, including sensor data, timestamp, container identifier, current voltage and the value of the opening counter.

- An unique code assigned to this particular user.

- The physical intensity of the signal received from the container.

- The current GPS coordinates of the user.

This information allows the server to associate this user with this particular container usage event. The intensity of the signal received is a method to distinguish between different users that can receive the same packet from the container. If several instances of the App running on different phones retransmit the same packet, the cloud-based server will assign the usage of the container to the user that has received the packet with the highest intensity, that is, the nearest one.

The opening counter, that takes account of the number of times the container lid has been opened, is an useful value for several reasons. First, it gives an estimation of the average volume of the waste being disposed, simply by dividing the capacity of the container by the number of times the container lid has been opened between two consecutive emptyings. Note that it is not necessary to reset the STERLING device when emptying the container, since the following measure will then return a greater distance between the ceiling and the waste level. In fact, it would be enough to open the lid once the container has been emptied: A STERLING App installed in the phone of the driver of the recycling truck would acquire this information automatically.

An additional use of the opening counter is to estimate the penetration of the STERLING App among citizenship. In many cases the Bluetooth packet emitted by the container will be simply lost, because there will be nobody with a STERLING App within its operational range (30 to 50 meters). By including the value of the opening counter, it is possible to estimate the number of packets being lost, thus obtaining an estimation of the use of the STERLING App by their final users. 


\section{UNDERSTANDING USERS' HABITS AND ENCOURAGING RECYCLING}

Our proposal aims not only to be a convenient way to avoid communication and power costs of the sensor system, but also to learn more about the usage of recycling containers, and to reward users, thus encourage recycling.

Regarding the data collection about recycling habits of the citizenship, the STERLING framework allows to obtain the following information:

- An estimation of the average volume of the waste being disposed, using the relationship between the number of times the container has been opened between two consecutive emptying operations.

- The times when the containers are used, as indicated by the corresponding timestamps. Many city councils define a specific time frame for waste disposal. To know the real times may be used to guide campaigns to encourage proper usage.

- Depending on the information collected by the STERLING App from users, additional segmentation by gender, age, or physical location can be carried out.

- Obviously, to know the fill level of the containers is a very useful information that can guide the paths of the trucks that empty them. Moreover, to properly detect and empty a full container has a positive impact in the surrounding area, avoiding waste to be disposed elsewhere.

- To know how frequently containers are full can also be used to adjust the number of containers in a particular area and/or the number of trucks needed, thus improving the logistics of waste collection.

- Finally, to know the fill levels of isolated containers in small villages avoids the need of sending a recycling truck only to check that the container is still half empty. For this particular case, a version of the STERLING sensor system with a SIM card may be more useful than the Bluetooth version, since the use of these containers in towns with low population density is expected to be scarce, so as the number of users of the STERLING App.

The STERLING framework has been designed to reward users for their recycling habits. The framework only takes accounting of how many times a particular user has opened the container lid. To avoid fraud, it is enough to truncate to a maximum value the number of daily uses of a container for a single user.

The different rewarding mechanisms and their advantages and drawbacks are beyond the scope of this paper. We can imagine different rewarding methods: From points or cash vouchers that can be exchanged for goods of any kind, to reductions in the taxes collected from the City Councils related to waste management. Moreover, knowing the location of the citizens that make use of the containers allows to establish a competition between neighborhoods or cities, that is another way of introducing gamification in the equation.

\section{CONCLUSIONS AND FUTURE WORK}

IoT is a disruptive technology that allows to improve many logistic processes. In this paper we propose STERLING, a framework that links recycling activities with their users, allowing to establish individual and collective rewarding systems while reducing operational costs of sensor systems installed in containers. Both objectives are accomplished by eliminating the need of a modem-based Internet connection, with the consequent reduction in communication costs and energy needed for its operation. Our current and future work includes the definition of the most appropriate rewarding mechanism with the help of interested agents (city councils, container manufacturers, recycling plants, and organizations that promote recycling), in order to launch a pilot to verify the usefulness of this framework.

\section{ACKNOWLEDGMENTS}

This research has been partially supported by MICINN (Spain) and ERDF program of the European Union: PCAS project (TIN2017-88614-R), CAPAP-H6 Network (TIN2016-81840-RED), and Junta de Castilla y Leon FEDER Grant VA082P17 (PROPHET Project). The authors would also like to thank José Luis Moreno, Jesús Aguirre and Zacarías Torbado, from ECOEMBES, for several inspiring conversations on this topic. 


\section{REFERENCES}

[1] Satori, M., Megantara, E. N., Primiana, I. F., and Gunawan, B., "Review of the influencing factors of integrated waste management," INTERNATIONAL JOURNAL OF GEOMATE 15(48), 34-40 (2018).

[2] Bluetooth SIG, I., "Bluetooth core version 4.2." https://ww.bluetooth.com/specifications/ adopted-specifications (2014). Accesed: 25-05-2017.

[3] Giacobbe, M., Puliafito, C., and Scarpa, M., "The big bucket: An iot cloud solution for smrt wste mngement in smrt cities," in [European Conference on Service-Oriented and Cloud Computing], 43-58, Springer (2016).

[4] Lindström, J., Hermanson, A., Hellis, M., and Kyösti, P., "Optimizing recycling management using industrial internet supporting circular economy: a case study of an emerging ips2," Procedia CIRP 64, 55-60 (2017).

[5] Chin, J. and Callaghan, V., "Recyclable, eco-friendly, on-demand bin (redbin)," in [Intelligent Environments (IE), 2014 International Conference on], 222-225, IEEE (2014).

[6] Gattim, N. K., Krishna, M. G., Nadh, B. R., Madhu, N., and Reddy, C. L., "Iot-based green environment for smart cities," in [Microelectronics, Electromagnetics and Telecommunications], 263-271, Springer (2018).

[7] Bharadwaj, B., Kumudha, M., Chaithra, G., et al., "Automation of smart waste management using iot to support swachh bharat abhiyan-a practical approach," in [Computing and Communications Technologies (ICCCT), 2017 2nd International Conference on], 318-320, IEEE (2017).

[8] Shea, T., [Gamification: Using gaming technology for achieving goals], The Rosen Publishing Group (2013).

[9] Briones, A. G., Chamoso, P., Rivas, A., Rodríguez, S., De La Prieta, F., Prieto, J., and Corchado, J. M., "Use of gamification techniques to encourage garbage recycling. a smart city approach," in [International Conference on Knowledge Management in Organizations], 674-685, Springer (2018).

[10] Berengueres, J., Alsuwairi, F., Zaki, N., and Ng, T., "Gamification of a recycle bin with emoticons," in [Proceedings of the 8th ACM/IEEE international conference on Human-robot interaction], 83-84, IEEE Press (2013).

[11] Janson, M., The Gamification of Recycling Behaviour, PhD thesis, University of Waikato (2016).

[12] SIMCom Wireless Solutions Ltd, "Sim800 series_at command manual_v1. 09," (august 2015). https:// www.elecrow.com/wiki/images/2/20/SIM800_Series_AT_Command_Manual_V1.09.pdf. 